\title{
Bienestar psicológico y burnout en personal de salud durante la pandemia de COVID-19
}

\section{Psychological well-being and burnout in health care providers during the COVID-19 pandemic}

\author{
Michelle García-Torres, Antonio de Jesús Aguilar-Castro y Mirna García-Méndez \\ Universidad Nacional Autónoma de México, México
}

\begin{abstract}
Resumen
El objetivo de la presente investigación fue evaluar a médicos/as y enfermeros/as respecto a su bienestar psicológico y burnout experimentado por la pandemia de la COVID-19 en México. Participaron 121 personas voluntarias, 56 médicos/as y 65 enfermeros/as, con un rango de edad de 22 a 67 años. Los resultados mostraron una baja prevalencia del síndrome de burnout. En bienestar psicológico, se encontraron diferencias por género, puntuando más alto los hombres que las mujeres en autoaceptación y propósito de vida. Respecto al sector donde se trabaja, los empleados del sector privado reportan mayor crecimiento personal, que los del sector público. En cuanto al burnout, se encontraron diferencias entre médicos/as y enfermeros/as en el factor despersonalización, puntuando más alto los médicos/médicas. En las comparaciones por sector, los que trabajan en un contexto privado reportaron mayores niveles de realización personal, que los pertenecientes a uno público. Compraraciones de grupo entre aquellos participantes contagiados por la COVID-19 en algún momento de la pandemia y los que no, mostraron que el grupo de contagio puntuó con mayor cansancio emocional y menor realización personal.
\end{abstract}

Palabras clave: bienestar psicológico, burnout, médicos, enfermeros, COVID-19

\begin{abstract}
The aim of this research was to evaluate physicians and nurses, men and women, in terms of their psychological wellbeing and burnout experienced during the COVID-19 pandemic in Mexico. Participants included 121 volunteers, 56 physicians and 65 nurses, between the ages of 22 and 67 . The results show a low prevalence of burnout syndrome. For psychological well-being, differences were found by gender, with men scoring higher in self-acceptanc and life purpos than women. As regards the sector of employment, employees in the private sector reported greater personal growth than those in the public sector. As to burnout, differences were found between physicians and nurses in the depersonalization factor, with physicians scoring higher. Comparisons by sector showed that those who work in a private context reported higher levels of personal fulfillment, than those that belong to a public one. Group comparisons between those participants who have had a COVID-19 infection at some point during the pandemic and those who have not, showed that the contagion group scored higher in emotional fatigue, and lower in personal fulfillment.
\end{abstract}

Keywords: psychological well-being, burnout, physicians, nurses, COVID-19

Como citar: García-Torres M., Aguilar-Castro A. J., \& García-Méndez M. (2021). Bienestar psicológico y burnout en personal de salud durante la pandemia de COVID-19. Escritos de Psicología - Psychological Writings, 14(2), 96-106. https:/l doi.org/10.24310/espsiescpsi.v14i2.13618

Correspondencia: Michelle García-Torres. Universidad Nacional Autónoma de México, MÉXICO. E-mail: michelle.garcia@ zaragoza.unam.mx E-mail del coautor Antonio de Jesús Aguilar Castro: jesus94.ac@gmail.com E-mail de la coautora Mirna García Méndez: mina@unam.mx 


\section{Introducción}

La pandemia de COVID-19 ha generado estrés, preocupación y miedo en la población en general, debido al rápido aumento de contagios y a la desinformación. Los problemas psicológicos y mentales han aumentado por el distanciamiento y aislamiento social (Juárez-García, 2020). La mayoría de las investigaciones enfocadas en el contexto de la COVID-19 se han delimitado en las características clínicas y epidemiológicas de la enfermedad, la estructura del virus SARS-CoV-2 y las políticas de salud pública para enfrentar la pandemia. Pero es menester examinar la salud mental de la población en general, la cual ha sido perjudicada en diversos sectores de la sociedad, en especial del personal de la salud (PS), quienes trabajan de cara a los pacientes con COVID-19.

Médicos y enfermeros de ambos sexos se encuentran en riesgo de complicar su estado de salud mental, en tanto estén sometidos a una considerable carga de trabajo y no reciban apoyo temprano de sus colegas, lo que puede derivar en desestabilidad emocional (Torres-Muñóz et al., 2020). El PS, debido a diversos factores y en las distintas etapas de la pandemia, puede presentar síntomas depresivos, ansiosos y similares al trastorno de estrés postraumático, afectando su atención, toma de decisiones y su bienestar (Huarcaya-Victoria, 2020).

La pandemia de COVID-19 ha puesto en el PS una enorme presión de trabajo que puede causar un agotamiento físico y mental, pero, además, los ha expuesto a un alto riesgo de infectarse, ser discriminados, aislarse, entre otras cosas, que causan problemas de salud mental como estrés, ansiedad, síntomas depresivos, insomnio, negación, ira y temor (Lozano-Vargas, 2020). En un estudio realizado en Colombia por Monterrosa-Castro et al. (2020) se muestra que el PS ha sufrido algún tipo de discriminación durante la pandemia por COVID-19, lo que puede estar relacionado a mayores alteraciones emocionales. Se reporta que más del $70 \%$ de las personas evaluadas manifiestan tener miedo de padecer COVID-19, ser fuente de contagio para sus familiares, ser portadores asintomáticos e incluso morir.

EI PS reporta múltiples formas de estrés, desde traumas asociados con las muertes de sus pacientes por COVID-19, ansiedad asociada con la inusual y demandante carga de trabajo, nuevas prácticas médicas y daño moral (Liberati et al., 2021). Lo que, al combinarse con la experiencia vivida de aislamiento y privación, aumenta los problemas de índole psicológica como el dolor, la soledad, la angustia y el daño moral. Para Deb et al. (2020) la salud mental está relacionada de manera positiva con el bienestar. Las personas con buena salud mental están satisfechas con sus vidas, encuentran un sentido de vida, son optimistas y tienen el coraje de resistir etapas críticas en la vida. Si una persona interpreta una situación o dificultad de manera positiva, permanecerá menos vulnerable a las alteraciones mentales y posiblemente se sentirá más feliz.

\section{Bienestar psicológico en PS}

En las últimas décadas se han realizado estudios que abordan el bienestar desde dos grades tradiciones: la primera con un enfoque en la felicidad o bienestar hedónico y la segunda ligada al desarrollo del potencial humano o bienestar eudaimónico (Ryan \& Deci, 2001). La primera tradición correspondería al bienestar subjetivo, mientras que la segunda al bienestar psicológico, que refiere al conjunto de la felicidad subjetiva, el significado de la vida y la satisfacción con esta (Deb et al., 2020).

Ryff y Keyes (1995) mencionan seis componentes del bienestar psicológico, que incluyen: evaluaciones positivas del sí mismo y de la vida pasada, autoaceptación; un sentido de crecimiento continuo y desarrollo como persona, crecimiento personal; creencia de que la vida propia tiene un propósito y significado, propósito de vida; poseer relaciones de calidad con otros, relaciones positivas con otros; la capacidad de manejar de manera efectiva la vida propia y el medio que le rodea, dominio del medio; y un sentido de autodeterminación, autonomía. En un estudio realizado por Andleeb et al. (2020) en Pakistán, se encontró que el bienestar psicológico está relacionado de manera negativa con la ideación suicida y con la angustia psicológica.

Al estudiar el bienestar psicológico en PS durante la pandemia de COVID-19, Fattori et al. (2021) señalan que el ser mujer, enfermera, joven y estar directamente involucrada en áreas de trabajo de COVID-19 o el tener un familiar infectado, son quienes presentan menores niveles de bienestar psicológico en comparación con sus colegas. Se enfatiza que los factores de riesgo potenciales para experimentar malestar psicológico pueden ser características preexistentes como el género, el rol ocupacional y las condiciones médicas previas.

Un estudio realizado por Veliz et al. (2018) en Chile con profesionistas sanitarios, mostró que son las mujeres quienes presentan un nivel bajo de bienestar psicológico, con mayor insatisfacción en los niveles de relaciones positivas, autonomía y crecimiento personal. Se menciona que la sobrecarga de trabajo puede influir en las relaciones que se establecen con los compañeros y por ende en su bien- 
estar y felicidad. En el caso particular de Irán, también se han encontrado diferencias en el bienestar psicológico de hombres y mujeres (Sharbafshaaer, 2019). Se reporta que quienes presentan mayores niveles de dominio del medio, relaciones positivas, propósito de vida y autoaceptación son los hombres.

EI PS que trabaja en la primera línea de atención a pacientes con COVID-19, especialmente médicos, médicas, enfermeros y enfermeras, perciben una expectativa que va más allá de su rol para hacer que las cosas se mantengan a flote (Liberati et al., 2021). Lo que, acompañado de una falta de reconocimiento de su labor, puede conducir a altos niveles de estrés. Saavedra-Macías et al. (2020) mencionan que en algunos casos el PS ha interiorizado la imagen de que son elementos portadores de peligro para su familia, amigos o personas cercanas, lo que afecta de manera negativa su bienestar subjetivo y aumenta la probabilidad de sufrir ansiedad y depresión.

Cuando los profesionales sanitarios se sienten satisfechos intrínsecamente con su trabajo, su bienestar psicológico aumenta (López-García et al., 2018). Se enfatiza que la satisfacción laboral se relaciona con la capacitación continua y la experiencia en el trabajo. Arrogante y Aparicio-Zaldivar (2019) señalan que un ambiente adverso en el lugar de trabajo puede afectar principalmente la autoa- ceptación experimentada por el personal, además de otras áreas del bienestar psicológico. También se ha reportado que aquellos que han sufrido de agresión psicológica por parte de las autoridades hospitalarias, poniendo en riesgo su estabilidad laboral, pueden experimentar miedo, temor, ansiedad y depresión (Bedoya, 2020).

\section{Burnout en PS}

La palabra burnout es un término anglosajón que refiere a estar quemado, desgastado, exhausto, lo que puede implicar una pérdida de la ilusión por la actividad laboral que se realiza (Aranda, 2006). Es un estado de agotamiento semejante a un fuego que se sofoca o una vela que se extingue (Schaufeli \& Buunk, 2003). Para Maslach y Jackson (1986) el síndrome de burnout es un fenómeno psicosocial resultado de un estrés crónico característico de la cotidianeidad del trabajo, que se compone de agotamiento emocional, despersonalización y una baja realización personal.

El estrés sostenido puede dar paso al síndrome de burnout, que se manifiesta con agotamiento emocional, síntomas físicos, despersonalización, sensación de ineficacia y pérdida de habilidades para la comunicación (Valdivieso et al., 2021). La Organización Mundial de la Salud, en su Clasificación Internacional de Enfermedades-11 (CIE-11, 2021) conceptualiza el burnout como un fenómeno ocupacional, un síndrome que es el resultado del estrés crónico por el lugar de trabajo, que no ha sido manejado adecuadamente.

Las personas que sufren burnout experimentan agotamiento, distanciamiento mental del propio trabajo, sentimientos negativos o cínicos respecto al empleo y una disminución en la autoeficacia profesional. Cardoso y Freire (2017) señalan que el burnout puede estar acompañado por síntomas físicos y psicológicos como cansancio, problemas de sueño y apetito, dolor físico, pesimismo, indiferencia, irritabilidad e indecisión. Arrogante y Aparicio-Zaldivar (2019) mencionan que el burnout puede tener un impacto negativo en la salud física y el bienestar. Específicamente la fatiga emocional puede ser predictora de problemas de salud física y mental por lo que se le ha considerado el primer paso para el desarrollo del síndrome de burnout.

Las principales complicaciones a las que están expuestos los profesionales de la salud son el síndrome de burnout y al virus SARS-CoV-2, causante de la enfermedad COVID-19. Lo que puede provocar que los individuos estén en crisis, afectándolos a nivel cognitivo, emocional y fisiológico, e interviniendo en su comportamiento y toma de decisiones (Macaya \& Aranda, 2020). En un estudio realizado por AlJhan et al. (2021) se encontró que el burnout fue detectado entre médicos y enfermeras durante la pandemia de COVID-19, pero que las enfermeras fueron las más afectadas. Cuando se realizaron comparaciones por años de experiencia, se encontró que el PS con menos años laborables tiene mayores probabilidades de presentar burnout.

EI PS, en su mayoría de enfermería y residentes, son quienes presentan niveles más altos de ansiedad, depresión y estrés, que van de severo a extremadamente severo, en comparación con el personal que no labora en áreas destinadas a la COVID-19. Lo que puede dar lugar al síndrome de burnout o a agravar trastornos como la depresión y ansiedad por factores como una mayor carga laboral, la infraestructura del lugar donde se labora y la protección contra la enfermedad (Martínez et al., 2020). El personal que trabaja en exceso puede sufrir de burnout, lo que eventualmente puede llevara una depresión (Halla et al., 2016). Se menciona que tanto la depresión como el burnout pueden manifestarse a través de fatiga, irritabilidad y una reducción en las funciones cognitivas, lo que puede crear un ambiente de trabajo bajo presión, con menor calidad en el cuidado de los pacientes, lo que puede colocar al PS en un riesgo mayor de cometer errores. 
Alkhamees et al. (2021) en su estudio realizado en Arabia Saudita con PS en pandemia de COVID-19, encontraron que aquellos que sufren de burnout tienen 8.88 más probabilidad de presentar síntomas de depresión. Se menciona que los factores que contribuyen a presentar mayores niveles de burnout se encuentra el ser soltero o divorciado, ser residente de los primeros dos años en la especialidad médica y el haber recibido ayuda en salud mental en los últimos dos años.

Entre el $17 \%$ y el $76 \%$ del personal médico cuenta con cifras muy elevadas de agotamiento emocional y son las mujeres quienes presentan con mayor frecuencia el síndrome de burnout (Graue et al., 2007). Esto debido a que perciben mayores presiones familiares del cónyuge. En el caso particular de México, un estudio realizado por Terrones et al. (2016) con médicos de distintas especialidades, mostró que la despersonalización fue donde se observó mayor afectación, seguida del agotamiento emocional y por último la realización personal. Al realizar comparaciones por género, los autores señalan que las mujeres reportaron mayores niveles de cansancio emocional, mientras que los hombres puntuaron más alto en despersonalización y realización personal.

Se ha reportado que el personal médico y de enfermería presentan ansiedad e insomnio, principalmente las mujeres (Pérez et al., 2020). Además de un deterioro en la satisfacción con las actividades cotidianas, en la confianza y fe en sí mismos, miedo a las responsabilidades, sensación de enfermedad, dolores de cabeza y cansancio generalizado. Amutio et al. (2008) señalan que, a mayores niveles de burnout, menores de bienestar psicológico. Se estima que la sobrecarga laboral predice el cansancio emocional y un bajo bienestar psicológico. También el deficiente desarrollo profesional, la falta de reconocimiento y poca supervisión del desempeño (coordinación organizacional) se relacionan con un alto índice de burnout.

\section{Bienestar psicológico y burnout en PS}

El burnout y el bienestar del PS está relacionado con la seguridad de los pacientes. Un bienestar deficiente, caracterizado por depresión, ansiedad, pobre calidad de vida y estrés, además de altos niveles de burnout, están significativamente asociados con un mayor número de auto errores reportados (Halla et al., 2016). Mientras que el bienestar psicológico puede ser protector contra el burnout ya que puede mitigar sus efectos negativos como los pensamientos de abandono de la profesión, una vida social y familiar restringida y afecciones en la salud física y psicológica (Amutio et al., 2008).

Entre las recomendaciones para mejorar la salud mental del personal de salud se encuentran las estrategias de relajación, el apoyo social y contención emocional de parte de la familia y colegas, tiempo de recreación, control del consumo de alcohol y la asistencia laboral por parte del equipo de trabajo (Torres-Muñóz et al., 2020). Mientras que Arrogante y Aparicio-Zaldivar (2019) mencionan que para reducir el síndrome de burnout en el PS se debe tener como principal objetivo mejorar sus niveles de autoaceptación a través del aumento de su autoestima.

Las investigaciones anteriores resaltan la necesidad de prestar atención a la salud mental del PS debido a que son la primera línea de combate de la pandemia de COVID-19. Es así como el objetivo general de esta investigación fue evaluar a médicos/médicas y enfermeros/enfermeras respecto a su bienestar psicológico y burnout experimentado por la pandemia de COVID-19 en México. Como objetivos específicos se busca comparar a médicos/médicas y enfermeros/enfermeras respecto a las dos variables bajo estudio, así como realizar comparaciones por género, sector donde se labora y entre aquellos que a lo largo de la pandemia se han contagiado de COVID-19 y los queno.

\section{Participantes}

Método

Se contó con la participación de 121 personas voluntarias. Dentro de los criterios de inclusión se consideró que fueran profesionales de la salud, médicos/médicas y enfermeros/enfermeras que estuvieran laborando durante la pandemia. Se incluyó a participantes del sector público y privado. Se excluyó del estudio al PS sin acceso a internet, ya que la aplicación se realizó por medios digitales.

La muestra quedó conformada por 56 médicos/médicas y 65 enfermeros/enfermeras, 38 hombres y 83 mujeres, con un rango de edad de 22 a 67 años, $(M=35.31, D E=10.86)$. El $46.6 \%$ pertenecientes a la ciudad de México, el 39.8\% al Estado de México y el 13.6\% del interior de la República. El 50.4\% reportó ser soltero, el $33.1 \%$ casado, el $7.4 \%$ vivir en unión libre, $6.6 \%$ estar separado o divorciado y el $2.5 \%$ ser viudo. Respecto al sector en el que se labora, el $76.9 \%$ pertenece al sector público y el $23.1 \%$ al privado. Entre los participantes se reportó tener jornadas laborales de 7 a 92 horas semanales $(M=$ 38.23, $D E=18.26$ ). El $45.5 \%$ reportó trabajar en atención de primera línea a pacientes con COVID-19 durante la pandemia, no así el 54.5\%. De la muestra el $43 \%$ reporta haberse contagiado de COVID-19 en algún momento desde el inicio de la pandemia, mientras que el $57 \%$ no reportó haberse contagiado. 


\section{Instrumentos}

Bienestar psicológico. Se aplicó la adaptación española de las escalas de bienestar psicológico de Ryff, de Díaz et al. (2006). La escala se compone por 29 reactivos, como "Cuando repaso la historia de mi vida estoy contento con cómo han resultado las cosas", "Siento que mis amistades me aportan muchas cosas" y "En su mayor parte, me siento orgulloso de quien soy y la vida que llevo", distribuidos en seis factores que son: autoaceptación, relaciones positivas, autonomía, dominio del entorno, propósito en la vida y crecimiento personal. El formato de respuesta utiliza una escala tipo Likert con puntuaciones comprendidas entre 1 (totalmente en desacuerdo) a 6 (totalmente de acuerdo). El Alpha de Cronbach reportada para el presente estudio fue de 0.86 .

Burnout. Se aplicó el Maslach Burnout Inventory, en su versión validada para población mexicana por Aranda et al. (2016). El instrumento se compone de 22 reactivos, como "Debido a mi trabajo me siento emocionalmente agotado", "Creo que trato a algunos pacientes como si fueran objetos" y "En el trabajo siento que estoy al límite de mis posibilidades", divididos en tres factores: agotamiento o cansancio emocional, despersonalización y realización personal. La escala evalúa la frecuencia con la que se experimentan las afirmaciones planteadas, en un continuo de 0 (nunca) a 6 (siempre). El instrumento detecta el burnout al sumar los puntajes obtenidos: para el cansancio emocional puntuaciones mayores a 26 puntos (con un puntaje máximo de 54); para la despersonalización puntuaciones mayores a 9 (con un puntaje máximo de 30); y en el caso de la realización personal puntajes menores a 34 (con un máximo de 48). La escala presenta un Alpha de Cronbach de 0.65.

\section{Procedimiento}

Se distribuyó una encuesta online a través de redes sociales dirigida a médicos/médicas y enfermeros/ enfermeras de todas las especialidades y niveles. Se contactó a PS del "Hospital General Dr. Rubén Leñero", de la "Clínica de Especialidades Churubusco", del "Centro Médico Nacional Siglo XXI" y del "Hospital General La Raza" a quienes se les invitó a participar en el estudio, así como a distribuirlo entre sus colegas también de la práctica privada.

La encuesta comprendía tres elementos: cuestionario sociodemográfico, la escala de bienestar psicológico y el instrumento que evalúa el burnout. La encuesta se realizó de manera online usando una plataforma de uso común, Google Forms. Se informó a todos los participantes sobre los objetivos del estudio y se proporcionó un consentimiento informado, asegurando la confidencialidad y el anonimato de la información.

La presente investigación es considerada sin riesgo (categoría I). Entendiendo por "riesgo de la investigación" la probabilidad de que los individuos que participan en la investigación sufran algún daño como consecuencia inmediata o tardía del estudio, de acuerdo con el Reglamento de la Ley General de Salud en Materia de Investigación para la Salud (1984), en su artículo 17.

\section{Análisis de datos}

Todos los análisis estadísticos se realizaron usando el paquete estadístico SPSS versión 25 . Se obtuvieron los estadísticos descriptivos de los participantes en los instrumentos aplicados. Se aplicó estadística paramétrica (prueba t-Student para muestras independientes) para el análisis del bienestar psicológico y estadística no paramétrica (U Mann-Whitney) para el estudio del burnout, así como una correlación de rango de Spearman para el análisis de la relación entre variables. También se realizaron análisis de regresión lineal múltiple para la predicción del burnout.

\section{Resultados}

La Tabla 1 muestra el promedio, la desviación estándar y el valor mínimo y máximo que obtuvo la muestra en los factores de los instrumentos de bienestar psicológico y burnout. La Tabla 2 contiene los estadísticos descriptivos por género, profesión, sector laboral y contagio de COVID-19 y la Tabla 3 muestra la prevalencia del burnout en los participantes de este estudio.

Respecto al bienestar psicológico, una prueba t-Student para muestras independientes no mostró diferencias estadísticamente significativas entre médicos/médicas y enfermeros/enfermeras. Al realizar comparaciones por género, se encontraron diferencias estadísticamente significativas en: el factor de autoaceptación $t(111)=2.69, p \leq 0.01$, puntuando los hombres más alto $(M=20.82)$ que las mujeres $(M=19.25) ; \mathrm{y}$ en el factor propósito de vida $t(118)=2.90, p \leq 0.01$, siendo nuevamente los hombres $(M$ $=26.50)$ quienes puntuaron más alto que las mujeres $(M=24.43)$. Al realizar comparaciones por sector donde se labora, se encontraron diferencias estadísticamente significativas en el factor crecimiento personal $t(47)=-2.04, p \leq 0.05$, puntuando más alto los que pertenecen al sector privado $(M=21.46)$, 
que al público $(M=20.00)$. No se encontraron diferencias estadísticamente significativas al realizar comparaciones entre aquellos que tuvieron COVID-19 con aquellos que no.

Tabla 1

Descriptivos del bienestar psicológico y burnout en médicos/médicas y enfermeros/enfermeras

\begin{tabular}{|c|c|c|c|c|}
\hline Variable & $M$ & $D E$ & Mínimo & Máximo \\
\hline Autoaceptación & 19.74 & 3.6 & 6 & 24 \\
\hline Relaciones Positivas & 21.83 & 5.17 & 9 & 30 \\
\hline Autonomía & 26.95 & 5.17 & 13 & 36 \\
\hline Dominio del entorno & 23.33 & 4.45 & 9 & 30 \\
\hline Propósito en la vida & 25.08 & 4.64 & 9 & 30 \\
\hline Crecimiento personal & 20.33 & 3.46 & 9 & 24 \\
\hline Cansancio Emocional & 17.88 & 9.14 & 1 & 49 \\
\hline Despersonalización & 4.49 & 4.21 & 0 & 18 \\
\hline Realización Personal & 33.56 & 6.97 & 16 & 48 \\
\hline
\end{tabular}

Tabla 2

Estadísticos descriptivos por género, profesión, sector laboral y contagio de COVID-19

\begin{tabular}{|c|c|c|c|c|c|c|c|c|c|c|c|}
\hline \multirow[t]{2}{*}{ Variables } & \multirow[t]{2}{*}{ Valores } & \multirow[b]{2}{*}{$N=121$} & \multicolumn{6}{|c|}{ Bienestar Psicológico } & \multicolumn{3}{|c|}{ Burnout } \\
\hline & & & Autoaceptación & $\begin{array}{l}\text { Relaciones } \\
\text { Positivas }\end{array}$ & Autonomía & $\begin{array}{c}\text { Dominio del } \\
\text { entorno }\end{array}$ & $\begin{array}{l}\text { Propósito en } \\
\text { la vida }\end{array}$ & $\begin{array}{c}\text { Crecimiento } \\
\text { personal }\end{array}$ & $\begin{array}{l}\text { Cansancio } \\
\text { emocional }\end{array}$ & $\begin{array}{c}\text { Despersona- } \\
\text { lización }\end{array}$ & $\begin{array}{c}\text { Realización } \\
\text { personal }\end{array}$ \\
\hline & & $\mathrm{n}(\%)$ & $M / D E$ & $M / D E$ & $M / D E$ & $M / D E$ & $M / D E$ & $M / D E$ & $\begin{array}{c}\text { Rango } \\
\text { promedio }\end{array}$ & $\begin{array}{c}\text { Rango } \\
\text { promedio }\end{array}$ & $\begin{array}{c}\text { Rango } \\
\text { promedio }\end{array}$ \\
\hline \multirow{2}{*}{ Género } & Mujer & $83(68.6)$ & $19.25 / \pm 3.97$ & $21.59 / \pm 5.34$ & $26.95 / \pm 5.31$ & $23.35 / \pm 4.65$ & $24.43 / \pm 5.20$ & $20.08 / \pm 3.54$ & 62.62 & 58.22 & 62.31 \\
\hline & Hombre & $38(31.4)$ & $20.82 / \pm 2.36$ & $22.37 / \pm 4.81$ & $26.95 / \pm 4.95$ & $23.29 / \pm 4.07$ & $26.50 / \pm 2.62$ & $20.89 / \pm 3.28$ & 57.46 & 67.07 & 58.14 \\
\hline \multirow{2}{*}{ Profesión } & $\begin{array}{l}\text { Médicos/ } \\
\text { médicas }\end{array}$ & $56(46.3)$ & $20.20 / \pm 3.84$ & $22.11 / \pm 5.17$ & $27.27 / \pm 5.05$ & $23.32 / \pm 4.58$ & $25.55 / \pm 4.77$ & $20.79 / \pm 4.01$ & 61.48 & 68.72 & 62.71 \\
\hline & $\begin{array}{l}\text { Enfermeros/ } \\
\text { enfermeras }\end{array}$ & $65(53.7)$ & $19.35 / \pm 3.38$ & $21.60 / \pm 5.21$ & $26.68 / \pm 5.31$ & $23.34 / \pm 4.39$ & $24.68 / \pm 4.53$ & $19.95 / \pm 2.89$ & 60.58 & 54.35 & 59.52 \\
\hline \multirow{2}{*}{$\begin{array}{l}\text { Sector } \\
\text { laboral }\end{array}$} & Público & $93(76.9)$ & $19.49 / \pm 3.75$ & $21.71 / \pm 5.04$ & $26.81 / \pm 5.09$ & $23.18 / \pm 4.53$ & $24.94 / \pm 4.77$ & $20.00 / \pm 3.46$ & 61.65 & 62.09 & 56.27 \\
\hline & Privado & $28(23.1)$ & $20.57 / \pm 2.99$ & $22.25 / \pm 5.67$ & $27.43 / \pm 5.51$ & $23.82 / \pm 4.26$ & $25.57 / \pm 4.22$ & $21.46 / \pm 3.28$ & 58.84 & 57.39 & 76.71 \\
\hline \multirow{2}{*}{ COVID-19 } & Sin contagio & $69(57)$ & $19.74 / \pm 3.75$ & $21.87 / \pm 5.16$ & $27.04 / \pm 5.35$ & $23.10 / \pm 4.76$ & $25.33 / \pm 4.44$ & $20.61 / \pm 3.48$ & 55.41 & 60.45 & 67.36 \\
\hline & \multicolumn{2}{|c|}{ Con contagio 52 (43) } & $19.75 / \pm 3.44$ & $21.79 / \pm 5.24$ & $26.83 / \pm 4.99$ & $23.63 / \pm 4.05$ & $24.75 / \pm 4.92$ & $19.98 / \pm 3.44$ & 68.42 & 61.73 & 52.56 \\
\hline
\end{tabular}

$M=$ media, $D E=$ desviación estándar

Tabla 3

Prevalencia del burnout entre médicos/médicas y enfermeros/enfermeras

\begin{tabular}{lcc}
\multicolumn{1}{c}{ Variables } & Frecuencia & Porcentaje \% \\
\hline Sindrome de Burnout $(n=121)^{\mathrm{a}}$ & 4 & 3.4 \\
Sí & 117 & 96.6 \\
No & & 16.5 \\
Subescalas del Burnout $(n=121)^{\mathrm{a}}$ & 20 & 11.6 \\
Cansancio Emocional Severo & 14 & 49.6 \\
Despersonalización Severa & 60 & 24 \\
Realización Personal Baja & & 25.6 \\
Subescalas del Burnout $(n=121)^{\mathrm{a}}$ & 29 & 31 \\
Cansancio Emocional Moderado & 34 & 28 \\
Despersonalización Moderada & 34 & \\
Realización Personal Media & & \\
\hline
\end{tabular}

a Determinado con base en las respuestas proporcionadas por los participantes, con niveles altos en las subescalas de cansancio emocional y despersonalización (ver sección instrumentos).

Respecto al burnout, una prueba U Mann-Whitney mostró diferencias estadísticamente significativas por profesión en el factor despersonalización $(p=0.024)$, presentando un rango medio superior los médicos (68.72) que los enfermeros (54.35). No se encontraron diferencias estadísticamente significativas por género. Al realizar comparaciones por sector donde se labora, se encontraron diferencias estadísticamente significativas en el factor realización personal $(p=0.007)$, presentando un rango medio superior los que trabajan en el sector privado (76.71) que en el público (56.27). En las comparaciones entre aquellos que en algún momento se han contagiado de COVID-19 con aquellos que no, se encontraron diferencias estadísticamente significativas en: el factor cansancio emocional $(p=0.043)$, con un rango medio superior el grupo que sí se ha contagiado (68.42) que el que no ha contraído 
COVID-19 (55.41); y en el factor realización personal ( $p=0.021)$, con un rango medio superior los participantes que no se han contagiado de COVID (67.36) frente a aquellos que sí (52.56).

Para conocer la relación entre variables, se realizó una correlación de rango de Spearman. Los resultados se muestran en la Tabla 4. Los datos muestran que los factores de cansancio emocional y despersonalización correspondientes a la variable burnout, correlacionan de manera negativa con todos los factores del bienestar psicológico evaluados. Mientras que el factor realización personal es el único que correlaciona de manera positiva con todos los factores del bienestar psicológico.

Con base en las correlaciones, se procedió a realizar análisis de predicción del burnout. Se realizaron pruebas de regresión lineal múltiple paso a paso, por profesión. En la Tabla 5 se muestran los resultados para el grupo de médicos/médicas y en la Tabla 6 los resultados de los enfermeros/enfermeras. Se reportan solo los resultados significativos. Se observa que para ambos grupos el dominio del medio es un predictor negativo del cansancio emocional, que confirma lo encontrado en las correlaciones. Respecto a la despersonalización, para el grupo de médicos/médicas el crecimiento personal es un predictor negativo, mientras que para los enfermeros/enfermeras solo la autonomía es predictora de tipo negativo. En la realización personal, para los médicos/médicas la autoaceptación y la autonomía son predictores positivos, mientras que para los enfermeros/enfermeras solo lo es la autonomía.

Tabla 4

Correlación Rho de Spearman para las variables bienestar psicológico y burnout

\begin{tabular}{|c|c|c|c|c|c|c|c|c|c|}
\hline & 1 & 2 & 3 & 4 & 5 & 6 & 7 & 8 & 9 \\
\hline 1. Autoaceptación & 1 & & & & & & & & \\
\hline 2. Relaciones Positivas & $.514^{\star *}$ & 1 & & & & & & & \\
\hline 3. Autonomía & $.496^{\star *}$ & $.500^{\star *}$ & 1 & & & & & & \\
\hline 4. Dominio del Entorno & $.581^{\star *}$ & $.537^{\star \star}$ & $.545^{\star \star}$ & 1 & & & & & \\
\hline 5. Propósito de Vida & $.773^{\star *}$ & $.434^{* *}$ & $.443^{\star *}$ & $.646^{* *}$ & 1 & & & & \\
\hline 6. Crecimiento Personal & $.514^{\star *}$ & $.486^{\star \star}$ & $.456^{\star *}$ & $.394^{\star *}$ & $.394^{* *}$ & 1 & & & \\
\hline 7. Cansancio Emocional & $-.300^{* *}$ & $-.395^{* *}$ & $-.347^{\star \star}$ & $-.410^{\star *}$ & $-.275^{\star *}$ & -.093 & 1 & & \\
\hline 8. Despersonalización & $-.217^{\star *}$ & $-.349^{* *}$ & $-.372^{* *}$ & $-.323^{* *}$ & $-.283^{* *}$ & $-.251^{\star *}$ & $.506^{* *}$ & 1 & \\
\hline 9. Realización personal & $.378^{\star *}$ & $.354^{* *}$ & $.477^{\star *}$ & $.365^{\star *}$ & $.356^{* *}$ & $.393^{* *}$ & $-.387^{\star *}$ & $-.426^{\star *}$ & 1 \\
\hline
\end{tabular}

$p<.05^{\star}, p<.01^{\star *}$

Tabla 5

Análisis de predicción del Burnout para el grupo de médicos/médicas

\begin{tabular}{|c|c|c|c|c|c|c|c|}
\hline Paso & Variable & $\mathrm{R}^{2}$ & $F$ & $B$ & $D E B$ & $\beta$ & $\Delta \mathrm{R}^{2}$ \\
\hline \multicolumn{8}{|c|}{ Cansancio emocional } \\
\hline 1 & Dominio del medio & $14 \%$ & $F(1,54)=8.65, p<.01$ & -.778 & .265 & -.372 & .14 \\
\hline \multicolumn{8}{|c|}{ Despersonalización } \\
\hline 1 & Crecimiento personal & $12 \%$ & $F(1,54)=7.61, p<.01$ & -.335 & .121 & -.351 & .12 \\
\hline \multicolumn{8}{|c|}{ Realización personal } \\
\hline 1 & Autoaceptación & $.29 \%$ & $F(1,54)=22.39, p<.01$ & 1.033 & .218 & .541 & .29 \\
\hline 2 & $\begin{array}{l}\text { Autoaceptación } \\
\text { Autonomía }\end{array}$ & $.40 \%$ & $F(2,53)=17.31, p<.01$ & $\begin{array}{l}.745 \\
.512\end{array}$ & $\begin{array}{l}.225 \\
.171\end{array}$ & $\begin{array}{l}.391 \\
.353\end{array}$ & .11 \\
\hline
\end{tabular}

$p<.05^{\star}, p<.01^{\star *}$

Tabla 6

Análisis de predicción del Burnout para el grupo de enfermeros/enfermeras

\begin{tabular}{cccccccc}
\hline Paso & Variable & $\mathrm{R}^{2}$ & $F$ & $B$ & $D E B$ & $\beta$ & $\Delta \mathrm{R}^{2}$ \\
\hline \multicolumn{7}{c}{} & \multicolumn{7}{c}{ Cansancio emocional } \\
\hline 1 & Dominio del medio & $32 \%$ & $F(1,63)=29.51, p<.01$ & -1.134 & .209 & -.565 & .32 \\
\hline & & Despersonalización & & & \\
\hline 1 & Autonomía & $13 \%$ & $F(1,63)=9.63, p<.01$ & -.308 & .099 & -.364 & .13 \\
\hline & & Realización personal & & \\
\hline 1 & Autonomía & $20 \%$ & $F(1,63)=15.57, p<.01$ & .561 & .142 & .445 & .20 \\
\hline
\end{tabular}

$p<.05^{\star}, p<.01^{\star *}$

\section{Discusión}

Los resultados de la presente investigación mostraron que la muestra estudiada de PS presenta niveles moderados de burnout. Respecto a la prevalencia de este, solo el 3.4\% reporta niveles severos de burnout. En las comparaciones entre médicos/médicas y enfermeros/enfermeras, no se encontraron diferencias en el bienestar psicológico, no así en el burnout. 
En el bienestar psicológico se encontraron diferencias por género en la autoaceptación y el propósito de vida, puntuando más alto los hombres. Esto indica que independientemente de la profesión de salud, son los hombres quienes tienen una evaluación más positiva de sí mismos y de su vida pasada hasta el momento actual, en comparación de las mujeres. Además, también son los hombres quienes en mayor grado consideran que su vida tiene un propósito y un significado, lo que puede proporcionarles protección contra los eventos estresantes de cada día. Estos hallazgos concuerdan con estudios como el de Fattori et al. (2021), Veliz et al. (2018) y Sharbafshaaer (2019), quienes señalan que los hombres puntúan más alto en bienestar psicológico que las mujeres, siendo el género un factor de riesgo para experimentar malestar psicológico.

Al realizar comparaciones por sector donde se labora, se encontró que el PS que trabaja en el sector privado puntúa más alto en el factor crecimiento personal, del bienestar psicológico, que los que laboran en el sector público. Lo que puede deberse a las carencias de equipo, falta de capacitación, la experiencia laboral (López-García et al., 2018), la falta de reconocimiento y poca supervisión del desempeño (Pérez et al., 2020), presentes en mayor proporción en el sector público.

En cuanto al burnout, se encontraron diferencias entre médicos/médicas y enfermeros/enfermeras en la despersonalización, puntuando más alto los médicos/médicas. Lo que implica que son estos quienes reconocen mayores niveles de frialdad, distanciamiento e insensibilidad hacia sus pacientes. Lo anterior coincide con lo mencionado por Terrones et al. (2016) cuando señala que la despersonalización es el área más afectada entre médicos de distintas especialidades. Pero las diferencias entre médicos/médicas y enfermeros/enfermeras difieren con lo encontrado por AlJhan et al. (2021) y Martínez et al. (2020) ya que mencionan que durante la pandemia de COVID-19, el grupo de enfermería es el más afectado por el burnout.

En las comparaciones por sector donde se labora, nuevamente se encontraron diferencias entre el PS que trabaja en el sector público y el privado, puntuando más alto estos últimos en el factor realización personal. Lo que implica que dentro del contexto privado el PS reporta mayores niveles de autoeficacia y realización laboral que dentro del contexto público. Lo que puede deberse a factores anteriormente mencionados como las carencias dentro de las instituciones públicas.

Los datos también mostraron diferencias en la escala de burnout entre el PS que durante la pandemia se ha contagiado de COVID-19 y aquellos que no. Los hallazgos muestran que el grupo de contagio presenta niveles más bajos de realización personal y más altos de cansancio emocional. Lo que puede implicar un mayor riesgo de sufrir el síndrome de burnout, ya que como lo mencionan Arrogante y Aparicio-Zaldivar (2019), el cansancio emocional es el primer paso para desarrollar el síndrome. Estos resultados resaltan la imperiosa necesidad de atender al PS que ha sufrido de COVID-19 ya que su salud física y mental pueden estar deterioradas lo que puede implicar un riesgo para la seguridad de los pacientes (Halla et al., 2016) ya que pueden cometer errores en su labor, debido a afecciones cognitivas, emocionales y fisiológicas (Macaya \& Aranda, 2020). Y verse mermado su bienestar en general, debido a que, a mayor presencia de burnout, menores niveles de bienestar psicológico (Amutio et al., 2008) y mayor riesgo de sufrir depresión (Alkhamees et al., (2021).

El análisis de correlación entre variables mostró que los factores de cansancio emocional y despersonalización de la escala de burnout correlacionan de manera negativa con todos los factores del bienestar psicológico. Lo que podría indicar que evaluaciones positivas del sí mismo y de la vida pasada, un sentido de crecimiento continuo y desarrollo como persona, la creencia de que la vida propia tiene un propósito y significado, poseer relaciones de calidad con otros, tener la capacidad de manejar de manera efectiva la vida propia y el medio que le rodea y un sentido de autodeterminación (Ryff \& Keyes, 1995), pueden ser factores protectores contra el síndrome de burnout. Lo anterior además se sustenta en los resultados de los análisis de predicción realizados.

En la muestra bajo estudio se encontró que el dominio del medio puede ser protector del cansancio emocional tanto de médicos/médicas como de enfermeros/enfermeras. Respecto al primer grupo, también se encontró que el crecimiento personal es protector de la despersonalización, mientras que para los enfermeros/enfermeras lo es la autonomía. También se reporta que la autoaceptación y la autonomía son predictores de la realización personal del grupo de médicos/médicas, mientras que solo lo es la autonomía para los enfermeros/enfermeras.

Los resultados de la presente investigación concuerdan con lo mencionado por Arrogante y Aparicio-Zaldivar (2019) y Torres-Muñóz et al. (2020), quienes indican que para mejorar la salud mental del PS y reducir el burnout son importantes las relaciones de apoyo con familiares y amigos, tener tiempo de recreación y mejorar la autoaceptación. 


\section{Limitaciones}

La presente investigación presenta limitaciones que tienen que ser consideradas al momento de interpretar los resultados. Una de ellas es que el estudio es de tipo transversal, lo que dificulta dar un seguimiento del bienestar psicológico y los niveles del burnout del PS en las distintas etapas de la pandemia e incluso antes de esta. Se sugiere para estudios futuros realizar aplicaciones y recogida de datos en los diferentes momentos de la crisis sanitaria y posteriores a esta. Otra limitación para considerar es que la muestra es reducida y participaron en su mayoría mujeres, lo que limita la generalización de los resultados. Además, es importante considerar el contexto geográfico y socioeconómico del estudio, ya que se realizó en participantes mayoritariamente de la ciudad de México y el Estado de México, pertenecientes a instituciones sanitarias de distintos estratos socioeconómicos, lo que puede significar una variación en los recursos con los que cuenta el PS para la atención de los pacientes. Se recomienda para estudios posteriores realizar la aplicación en distintos estados de la República Mexicana y considerar la variable socioeconómica en el estudio. Por último, pero no menos importante, se recomienda incluir en estudios posteriores a PS como auxiliares de enfermería, paramédicos, etc., quienes realizan una labor muy importante en la lucha contra el COVID-19.

\section{Conclusión}

El presente estudio enfatiza la imperiosa necesidad de atender y potenciar el bienestar psicológico del PS, en especial del género femenino, para así, como lo mencionan Amutio et al. (2008), mitigar los efectos negativos del burnout como son las ideas de querer abandonar la profesión, tener una vida familiar y social restringida, así como presentar problemas de salud a nivel físico y psicológico. Lo anterior teniendo en cuenta aspectos sociales y culturales de la muestra bajo estudio, ya que en el caso particular de México el PS femenino puede enfrentar demandas familiares (Graue et al., 2007) que afecten su desempeño laboral y bienestar psicológico.

Además, se resalta la necesidad de mejorar las condiciones laborales del PS que trabaja en el sector público, ya que las deficiencias en el medio de trabajo pueden contribuir a que se desarrolle el síndrome de burnout (Pérez et al., 2020). Esto teniendo en consideración el nivel socioeconómico donde se aplicó el estudio, ya que es conocimiento popular mexicano que con frecuencia la atención médica privada tiene mayores recursos tanto materiales como de personal que las instituciones públicas.

Los hallazgos también enfatizan la importancia de la atención a la salud mental del PS que ha sufrido de COVID-19 a lo largo de la pandemia, promoviendo su bienestar psicológico que les sirva de barrera contra los efectos adversos del cansancio emocional y los proteja de la despersonalización. Al mejorar la salud mental de las personas, se contribuye a que vivan vidas más satisfechas, con sentido, sean optimistas y puedan tener el coraje para resistir etapas críticas (Deb et al., 2020) como lo es la presente pandemia.

\section{Referencias}

1. Andleeb, S. N., Ahsan, S., \& Zaheer, S. (2020). Moderating Role of Psychological Distress on the Relationship Between Suicidal Ideation and Psychological Wellbeing among People with Physical Disabilities. Pakistan Armed Forces Medical Journal, 70(6), 1728-1733.

2. Alkhamees, A. A., Assiri, H., Alharbi, H. Y., Nasser, A., \& Alkhamees, M. (2021). Burnout and depression among psychiatry residents during COVID_19 pandemic. Human Resources for Health, 19(46), https://doi.org/10.1186/s12960-021-00584-1

3. AlJhani, S., AlHarbi, H., AlJameli, S., Hameed, L., AlAql, K., \& Alsulaimi, M. (2021). Burnout and coping among healthcare providers working in Saudi Arabia during the COVID-19 pandemic. Middle East Current Psychiatry, 28(29). https://doi.org/10.1186/s43045-021-00108-6

4. Aranda, C. (2006). Diferencias por sexo, síndrome de burnout y manifestaciones clíni- cas, en los médicos familiares de dos instituciones de salud, Guadalajara, México. Revista Costarricense de Salud Pública, 15(29), 1-7. https://www.scielo.sa.cr/scielo.php?script=sci arttext\&pid=S1409-14292006000200002

5. Aranda, C., Pando, M., \& Salazar, J. G. (2016). Confiabilidad y validación de la escala Maslach Burnout Inventory (Hss) en trabajadores del occidente de México. Salud Uninorte, 32(2), 218227.

6. Arrogante, O., \& Aparicio-Zaldivar, E. (2019). Burnout syndrome in intensive care professionals: Relationships with health status and wellbeing. Enfermería Intensiva, 31 (2, 60-70). https://doi. org/10.1016/i.enfi.2019.03.004 
7. Bedoya, C. M. (2020). Covid-19: La pandemia del maltrato contra el personal de la salud en tiempos de pandemia. Interdisciplinary Journal of Epidemiology and Public Health, 3(1). DOI: 10.18041/2665-427X/ijeph.1.6276ISSN: 2665-427X

8. Cardoso, B. R., \& Freire, I. C. J. (2017). Stress, burnout and coping in health professionals: a literature review. Psychological Brain Studies, 1(4), 1-8.

9. Deb, S., Thomas, S., Bose, A., \& Aswathi, T. (2020). Happiness, Meaning, and Satisfaction in Life as Perceived by Indian University Students and Their Association with Spirituality. Journal of Religion and Health, 59, 2469-2485. https://doi.org/10.1007/s10943-019-00806-w

10. Diario Oficial de la Federación. Reglamento de la Ley General de Salud en Materia de Investigación para la Salud. 1984. Disponible en:

11. http://www.salud.gob.mx/unidades/cdi/nom/compi/rlgsmis.html

12. Díaz, D., Rodríguez-Carvajal, R., Blanco, A., Moreno-Jiménez, B., Gallardo, I., Valle, C., \& Van Dierendonck, D. (2006). Adaptación española de las escalas de bienestar psicológico de Ryff. Psicothema, 18(3), 572-577. http://www.redalyc.org/articulo.oa?id=72718337

13. Fattori, A., Cantú, F., Comotti, A., Tombola, V., Colombo, E., Nava, C., Bordini, L., Riboldi, L., Bonzini, M., \& Brambilla, P. (2021). Hospital workers mental health during the COVID-19 pandemic: methods of data collection and characteristics of study sample in a university hospital in Milan (Italy). BMC Medical Research Methodology, 21(163). https://doi.org/10.1186/s12874-02101355-1

14. Graue, E., Álvarez, R., \& Sánchez, M. (2007). El síndrome de "Burnout": la despersonalización, el agotamiento emocional y la insatisfacción en el trabajo como problemas en el ejercicio de la medicina y el desarrollo profesional. [Seminario]. Universidad Nacional Autónoma de México. http://www.facmed.unam.mx/sms/seam2k1/2007/jun 01 ponencia.html

15. Hall, L. H., Johnson, J., Watt, I., Tsipa, A., O'Connor, D. B. (2016). Healthcare Staff Wellbeing, Burnout, And Patient Safety: A Systematic Review. PLOS ONE, 11(7). https://doi.org/10.1371/ journal.pone. 0159015

16. Huarcaya-Victoria, J. (2020). Consideraciones sobre la salud mental en la pandemia de COVID19. Revista Peruana de Medicina Experimental y Salud Pública, 37(2), 327-334 https://doi. org/10.17843/rpmesp.2020.372.5419

17. Juárez- García, A. (2020). Síndrome de burnout en personal de salud durante la pandemia COVID-19: un semáforo naranja en la salud mental. Salud UIS, 53(4), 432-439. https://doi. org/10.18273/revsal.v52n4-2020010

18. Kareaga, A., Ayestaran, A. \& Smith, J. (2008). Evaluación del burnout y bienestar psicológico en los profesionales sanitarios del país Vasco. Revista de Psicología del Trabajo y de las Organizaciones, 24(2), 235-252.

19. https://scielo.isciii.es/scielo.php?script=sci arttext\&pid=S1576-59622008000200006

20. Liberati, E., Richards, N., Williars, J., Scott, D., Boydell, N., Parker, J., Pinfold, V., Martin, G., Dixon-Woods, M., \& Jones, P. B. (2021). A qualitative study of experiences of NHS mental healthcare workers during the Covid-19 pandemic. BMC Psychiatry, 21(250). https://doi.org/10.1186/ s12888-021-03261-8

21. López-García, C., Ruiz-Hernández, J. A., Llor-Zaragoza, L., Llor-Zaragoza, P. \& Jiménez-Barbero, J. A. (2018). Violencia de los usuarios y el bienestar psicológico en los profesionales de la atención primaria de salud. Revista Europea de Psicología Aplicada al Contexto Jurídico, 10(2), 57-63. https://dx.doi.org/10.5093/ejpalc2018a6

22. Lozano-Vargas, A. (2020). Impacto de la epidemia del Coronavirus (COVID-19) en la salud mental del personal de salud y en la población general de China. Revista de Neuro-Psiquiatría, 83(1), 51-56 1609-7394-rnp-83-01-51.pdf (scielo.org.pe)

23. Macaya, P. \& Aranda, F. (2020). Cuidado y autocuidado en el personal de salud: enfrentando la pandemia COVID-19. Revista Chilena de Anestesia, 49(3), 356-62.

24. Martínez, F., Azkoul, M., Rangel, C., Sandia, I. \& Pinto, S. (2020). Efectos de la pandemia por COVID-19 en la salud mental de trabajadores sanitarios del estado Mérida, Venezuela. GICOS, 5(e2), 77-88. https://dialnet.unirioja.es/servlet/articulo?codigo=7674979

25. Maslach, C., \& Jackson, S. E. (1986). Maslach Burnout Inventory. Palo Alto, CA.: Consulting Psychologist Press.

26. Monterrosa-Castro,A., Dávila-Ruiz, R., Mejía-Mantilla,A., Contreras-Saldarriaga, J., Mercado-Lara, M., \& Flores-Monterrosa, C. (2020). Estrés laboral, ansiedad y miedo al COVID-19 en médicos generales colombianos. MedUNAB, 23(2), 195-213. https://doi.org/10.29375/01237047.3890 
27. Pérez, P. X., Noroña, D. R., \& Vega, V. (2021). Repercusión SARS-CoV-2 en salud mental y bienestar psicológico del personal Centro de Salud Huambalo 2020. Revista Scientific, 6(19), 243-262. https://doi.org/10.29394/Scientific.issn.2542-2987.2021.6.19.12.243-262

28. Ryan, R. M., \& Deci, E. L. (2001). To be happy or to be self-fulfilled: A review of research on hedonic and eudaemonic well-being. In S. Fiske (Ed.). Annual Review of Psychology (pp. 141166). Palo Alto, CA: Annual Reviews, Inc.

29. Ryff, C. D., \& Keyes, C. L. M. (1995). The structure of psychological well-being revisited. Journal of Personality and Social Psychology, 69, 719-727. https://doi.org/10.1037/0022-3514.69.4.719

30. Saavedra-Macías, F. J., Arias-Sánchez, S., Rangel-Alcudia, F. J. \& Murvartian, L. (2020). COVID19 y estigma por asociación en profesionales socio-sanitarios. Apuntes de Psicología, 38(3), 139-147. idUS - COVID-19 y estigma por asociación en profesionales socio-sanitarios

31. Sharbafshaaer, M. (2019). Correlation between dimensions of psychological well-being with life satisfaction and self-regulation. Journal of Fundamentals of Mental Health, 21(3), 160-166.

32. Schaufeli, W. B. \& Buunk, B. P. (2003). Burnout: An overview of 25 years of research and therorizing. In M. J. Schabracq, J. A. M. Winnubst \& C. L. Cooper (Eds.). The Handbook of Work and the Health Psychology. John Wiley \& Sons, Ltd.

33. Terrones, J. F., Cisneros-Pérez, V., \& Arreola-Rocha, J. J. (2016). Síndrome de burnout en médicos residentes del Hospital General de Durango, México. Revista Médica del Instituto Mexicano del Seguro Social, 54(2), 242-248. http://www.redalyc.org/articulo.oa?id=457745149016

34. Torres-Muñóz, V., Farias-Córtes, J. D., Reyes-Vallejo, L. A. \& Guillén Díaz-Barriga, C. (2020). Riesgos y daños en la salud mental del personal sanitario por la atención a pacientes con COVID19. Revista Mexicana de Urología, 80(3), 1-9. https://www.medigraphic.com/cgi-bin/new/ resumen.cgi?IDARTICULO=94412

35. Valdivieso, J. A., Noroña, D. R. \& Vega, V. (2021). Síndrome de burnout en personal de atención a urgencias médicas durante la pandemia de covid-19. Revista de Investigación Talentos, 8(1), 93-100. https://doi.org/10.33789/talentos.8.1.146

36. Veliz, A. L., Dörner, A. P., Soto, A. G., \& Arriaga, A. (2018). Bienestar psicológico y burnout en profesionales de atención primaria de salud en la región de Los Lagos, Chile. Acta Universitaria, 28(3), 56-64. DOI: https://doi.org/10.15174/au.2018.1895

37. World Health Organisation (2021). Burn-Out an "Occupational Phenomenon": International Classification of Diseases. Available online: https://www.who. int/mental health/evidence/burn-out/en/

RECIBIDO: 1 de octubre de 2021

MODIFICADO: 15 de noviembre de 2021 ACEPTADO: 1 de diciembre de2021 